\title{
Ethanol Exposure Regulates Gabra1 Expression via Histone Deacetylation at the Promoter in Cultured Cortical Neurons
}

\author{
John Peyton Bohnsack, Vraj K. Patel, and A. Leslie Morrow \\ Department of Pharmacology (J.P.B., A.L.M.), Department of Psychiatry (A.L.M.), and Bowles Center for Alcohol Studies \\ (J.P.B., V.K.P., A.L.M.), University of North Carolina, Chapel Hill, North Carolina
}

Received April 24, 2017; accepted August 8, 2017

\begin{abstract}
$\gamma$-Aminobutyric acid $A$ receptors $\left(G_{A B A}-R s\right)$ mediate the majority of inhibitory neurotransmission in the adult brain. The $\alpha 1$-containing $\mathrm{GABA}_{\mathrm{A}}-\mathrm{Rs}$ are the most prominent subtype in the adult brain and are important in both homeostatic function and several disease pathologies including alcohol dependence, epilepsy, and stress. Ethanol exposure causes a decrease of $\alpha 1$ transcription and peptide expression both in vivo and in vitro, but the mechanism that controls the transcriptional regulation is unknown. Because ethanol is known to activate epigenetic regulation of gene expression, we tested the hypothesis that ethanol regulates $\alpha 1$ expression through histone modifications in cerebral cortical cultured neurons. We found that class I histone deacetylases (HDACs) regulate ethanol-induced changes in $\alpha 1$ gene and protein expression as pharmacologic inhibition or knockdown of HDAC1-3 prevents the effects of
\end{abstract}

ethanol exposure. Targeted histone acetylation associated with the Gabra1 promoter using CRISPR (clustered regularly interspaced palindromic repeat) dCas9-P300 (a nuclease-null Cas9 fused with a histone acetyltransferase) increases histone acetylation and prevents the decrease of Gabra1 expression. In contrast, there was no effect of a mutant histone acetyltransferase or generic transcriptional activator or targeting P300 to a distant exon. Conversely, using a dCas9-KRAB construct that increases repressive methylation $(\mathrm{H} 3 \mathrm{~K} 9 \mathrm{me} 3)$ does not interfere with ethanol-induced histone deacetylation. Overall our results indicate that ethanol deacetylates histones associated with the Gabra1 promoter through class I HDACs and that pharmacologic, genetic, or epigenetic intervention prevents decreases in $\alpha 1$ expression in cultured cortical neurons.

\section{Introduction}

$\gamma$-Aminobutyric acid type A receptors $\left(\mathrm{GABA}_{\mathrm{A}}-\mathrm{Rs}\right)$ are $\mathrm{Cl}^{-}$ ion channels that mediate the majority of inhibitory neurotransmission in the adult brain. $\mathrm{GABA}_{\mathrm{A}}-\mathrm{Rs}_{\mathrm{s}}$ are usually heteropentamers with different subunits of the $\mathrm{GABA}_{\mathrm{A}}-\mathrm{R}$ conveying heterogeneity of function, localization, and pharmacology (Uusi-Oukari and Korpi, 2010). $\mathrm{GABA}_{\mathrm{A}}-\mathrm{R}$ expression is differentially regulated in different disease states, including alcohol withdrawal (Devaud et al., 1997), epilepsy (Lund et al., 2008), autism (Fatemi et al., 2009), depression (Poulter et al., 2008), and schizophrenia (Hoftman et al., 2015).

$\mathrm{GABA}_{\mathrm{A}}-\mathrm{Rs}$ have long been known to be involved in acute alcohol intoxication, dependence, and withdrawal symptoms (Kumar et al., 2009), with acute alcohol potentiating GABAergic function and chronic alcohol exposure leading to $\mathrm{GABA}_{\mathrm{A}}-\mathrm{R}$ hypofunction. Increasing $\mathrm{GABA}_{\mathrm{A}}-\mathrm{R}$ function via pharmacologic

This work was supported by the National Institutes of Health National Institute on Alcohol Abuse and Alcoholism [Grant P60-AA11605] (to A.L.M.) and the Bowles Center for Alcohol Studies.

https://doi.org/10.1124/jpet.117.242446. agents such as benzodiazepines is useful for treating the symptoms of acute alcohol withdrawal syndrome such as increased anxiety, seizure susceptibility, central and autonomic nervous system hyperexcitability, and tremor (also known as delirium tremens) (Amato et al., 2010). One issue with benzodiazepine treatment is that decreases in $\mathrm{GABA}_{\mathrm{A}}-\mathrm{R}$ expression occur (Uusi-Oukari and Korpi, 2010), suggesting the need to find alternative strategies for increasing $\mathrm{GABA}_{\mathrm{A}}-\mathrm{R}$ expression in acute alcohol withdrawal syndrome and other disease states where $\mathrm{GABA}_{\mathrm{A}}-\mathrm{R}$ expression is dysregulated.

The $\alpha 1$-containing $\mathrm{GABA}_{\mathrm{A}}-\mathrm{Rs}$ are the most abundant subtype in the adult brain and are down-regulated in both alcohol withdrawal (Devaud et al., 1997) and epilepsy (Lund et al., $2008)$ in rodents. Chronic ethanol (EtOH) exposure decreases Gabra1 transcript expression (Montpied et al., 1991; Devaud et al., 1995b), $\alpha 1$ protein expression (Devaud et al., 1997; Kumar et al., 2002; Cagetti et al., 2003), and $\mathrm{GABA}_{\mathrm{A}}-\mathrm{R}$ hypofunction (Morrow et al., 1988; Cagetti et al., 2004; Liang et al., 2004) that is associated with increased withdrawal symptoms such as increased seizure susceptibility (Devaud et al., 1995a), cross-tolerance to benzodiazepines (Cagetti

ABBREVIATIONS: ANOVA, analysis of variance; ChIP, immunoprecipitation; CREB, cAMP response element binding; CRISPR, clustered regularly interspaced palindromic repeat; DIV, days in vitro; $\mathrm{EtOH}$, ethanol; $\mathrm{GABA}_{\mathrm{A}}-\mathrm{R}, \gamma$-aminobutyric acid A receptor; HAT, histone acetyltransferase; HDAC, histone deacetylase; MC1568, (2E)-3-[5-[(1E)-3-(3-fluorophenyl)-3-oxo-1-propen-1-yl]-1-methyl-1H-pyrrol-2-yl]-N-hydroxy-2-propenamide; MS-275, N-(2aminophenyl)-4-[N-(pyridin-3-yl-methoxycarbonyl) aminomethyl]benzamide; PBS, phosphate-buffered saline; PCR, polymerase chain reaction; qPCR, quantitative polymerase chain reaction; RGPF966, (2E)- $N$-(2-amino-4-fluorophenyl)-3-[1-(3-phenyl-2-propen-1-yl)-1H-pyrazol-4-yl]-2-propenamide; SAHA, suberoylanilide hydroxamic acid; sgRNA, small-guide RNA; shRNA, short hairpin RNA; siRNA, small-interfering RNA; TSA, trichostatin A; UNC, University of North Carolina. 
et al., 2003; Liang et al., 2007), and tremor (Kralic et al., 2005) in rodents. A 4-hour EtOH exposure $(50 \mathrm{mM})$ in cerebral cultured cortical neurons mimics the changes in $\mathrm{GABA}_{\mathrm{A}}-\mathrm{R} \alpha 1$ expression that have been shown in chronic EtOH exposure and withdrawal models in vivo, and this system has been used to determine molecular mechanisms that control their function and expression (Kumar et al., 2010; Carlson et al., 2013). Ethanol exposure also diminishes $\mathrm{GABA}_{\mathrm{A}}-\mathrm{R}$ function in cortical cultured neurons, including zolpidem potentiation (Kumar et al., 2010) and changes in mini-inhibitory postsynaptic current characteristics (Werner et al., 2011), but the molecular mechanisms that regulate the decreases in Gabra1 transcription after EtOH exposure are still poorly understood.

Recent studies suggest that EtOH regulates gene expression through epigenetic pathways involving post-translational modifications on histone tails (Pandey et al., 2008; Wang et al., 2008; Warnault et al., 2013). Changes in post-translational modifications of histones allow for either permissive or prohibitive access for transcription factors and other key components of transcription machinery to bind to DNA to initiate transcription. Acetylation of histone tails is associated with increased or permissive transcription (Wang et al., 2008) and is facilitated by a class of enzymes called histone acetyltransferases (HAT) and removed by histone deacetylases (HDACs) (Wang et al., 2009). The HDAC family consists of four different classes, which have different regulatory roles, subcellular localization, and pharmacology (Haberland et al., 2009). The $\mathrm{Zn}^{+}$-dependent HDACs (classes I, II, and IV) are localized in the nucleus and cytoplasm and control a number of different cellular functions including gene transcription (Broide et al., 2007; Haberland et al., 2009; Wang et al., 2009). HDAC inhibitors have recently been suggested for the treatment of alcohol use disorders (Pandey et al., 2008; Warnault et al., 2013; Simon-O'Brien et al., 2015). Treatment with histone deacetylase inhibitors prevents $\mathrm{GABA}_{\mathrm{A}}-\mathrm{R}$ hypofunction in the ventral tegmental area after chronic EtOH exposure possibly through restoring $\alpha 1$ expression through either a trafficking or gene activating mechanism (Arora et al., 2013). Another study found that the HDAC inhibitors enhanced folding, trafficking, and function of $\alpha 1$ (A322D), an $\alpha 1$ mutation that causes epilepsy (Di et al., 2013). However, changes in histone acetylation associated with Gabra1 gene elements (i.e., promoter) after EtOH have not yet been identified, nor have the HDACs or HATs that may regulate changes in Gabra1 gene expression been elucidated.

Changes in histone acetylation have long been associated with changes in gene transcription; however, experiments using HDAC inhibitors found that HDAC inhibitors only regulate a small set of genes $(\sim 2 \%)$ via histone acetylation (Van Lint et al., 1996); therefore, until recently determining whether changes in histone acetylation are associated with a specific gene or corresponding regulatory elements was not possible. CRISPR Cas9 has recently become the premier tool for gene targeting and editing technology (Doudna and Charpentier, 2014) as the tool is highly selective for certain loci in the genome. A modified Cas9 (dCas9-P300) has been used to make epigenetic changes at specific loci by using gene targeting ability of Cas9 in combination with histone acetyltransferase capabilities of the HAT P300 (Hilton et al., 2015).

We used a cerebral cultured cortical neuron system to determine the molecular mechanism that drives decreases in Gabra1 expression after EtOH exposure. We report that EtOH decreases Gabra1 expression through histone deacetylation at the Gabra1 promoter that is facilitated by class I HDACs. Furthermore, we demonstrate that increasing histone acetylation at the Gabra1 promoter using dCas9-P300 prevents EtOH-induced decreases in Gabra1 expression. Identification of the epigenetic regulators that modulate Gabra1 expression could be potentially useful for development of therapeutics for the treatment of various disorders where Gabra1 expression is dysregulated.

\section{Material and Methods}

\section{Primary Cultured Cortical Neurons}

Cerebral cortices from mixed sex (male/female $~ 50 \%$ ) SpragueDawley rat pups $(\mathrm{pn}=0$ ) were isolated then seeded at $1,000,000$ neurons per well or 100,000 neurons per well and grown for 18 days in vitro (DIV 18) (Dulbecco's modified Eagle's medium $+\mathrm{B} 27+$ penicillin and streptomycin, $37^{\circ} \mathrm{C}, 5 \% \mathrm{CO}_{2}$ ) as described previously elsewhere (Bohnsack et al., 2016). On DIV 14 antibiotics were removed. All procedures were performed in compliance with guidelines specified by the Institutional Animal Care and Use Committee at the University of North Carolina (UNC) at Chapel Hill.

\section{Drug Exposure}

On DIV 18, trichostatin A (TSA, 500 nM; Tocris Bioscience, Bristol, United Kingdom), suberoylanilide hydroxamic acid (SAHA, $3 \mu \mathrm{M}$; Tocris Bioscience), (2E)- $N$-(2-amino-4-fluorophenyl)-3-[1-(3-phenyl-2propen-1-yl)- $1 H$-pyrazol-4-yl]-2-propenamide (RGPF966, $24 \mathrm{nM}, 80 \mathrm{nM}$; Cayman Chemical, Ann Arbor, MI), (2E)-3-[5-[(1E)-3-(3-fluorophenyl)-3oxo-1-propen-1-yl]-1-methyl-1 $H$-pyrrol-2-yl]- $N$-hydroxy-2-propenamide (MC1568, $1 \mu \mathrm{M}$; Cayman Chemical), or sodium butyrate (1 mM; Tocris Bioscience) or equivalent volume vehicle (100\% v/v dimethylsulfoxide) was added directly to the cell culture medium for 4 hours.

The TSA concentration ( $500 \mathrm{nM}$ ) was chosen based on preliminary experiments showing increases in H3KAc in cell culture (data not shown). The SAHA and sodium butyrate doses were chosen based on the manufacturers' recommendations. The RGFP966 and MC1568 doses were chosen based on specificity for HDAC3 and class II HDACs, respectively.

Ethanol (50 mM) was co-exposed with drugs (or vehicle) for 4 hours. Experiments were stopped by placing the cell-culture plates on ice; the cells were then washed twice with ice-cold Dulbecco's phosphatebuffered saline (DPBS), and then lysed in either TRIzol or $0.32 \mathrm{M}$ sucrose homogenization buffer. Subcellular fractionation of the cultured neurons was performed as previously described elsewhere (Bohnsack et al., 2016).

\section{RNA Isolation and Quantitative Polymerase Chain Reaction}

Neurons were rinsed with ice-cold PBS then homogenized in TRIzol (Ambion, Austin, TX), and the RNA was extracted and purified according to the manufacturer's instructions. The RNA was quantified and quality controlled using a Nanodrop (all 260/280, and 230/260 values $\geq 1.8$; Fisher Scientific, Fair Lawn, NJ). Two milligrams of purified RNA were reversed transcribed to a cDNA library using High Capacity RNA to DNA kit (Applied Biosystems, Foster City, CA) following the manufacturer's instructions.

DNA (10 ng per reaction) was then subjected to quantitative polymerase chain reaction (qPCR) analysis using TaqMan gene expression probes and Taq Gene Expression MasterMix. The reactions were run in duplicate on a StepOnePlus RT-PCR system (Applied Biosystems) using glyceraldehdyde-3-phosphate dehydrogenase (Gapdh) as a loading control (Bohnsack et al., 2016).

Data were analyzed using the $\Delta \Delta \mathrm{CT}$ method and expressed as fold control. The catalog numbers of the TaqMan probes are Gabra1, Rn00788315_m1; Hdac1, Rn01519308_g1; Hdac2, Rn01193634_g; Hdac3, Rn00584926_m1; and Gapdh, Rn01775763_g1. 


\section{Western Blot Analysis}

The Western blot analyses were performed as previously described elsewhere, with some modifications (Bohnsack et al., 2016). The blots were blocked for 1 hour (room temperature) in Li-Cor Blocking Buffer + PBS $(1: 1 \mathrm{v} / \mathrm{v})$. Primary antibodies were added to the blot in Li-Cor Blocking Buffer + PBST (1:1 v/v, 0.1\% Tween 20) (Li-Cor Biosciences, Lincoln, NE) and incubated overnight at $4^{\circ} \mathrm{C}$. The blots were washed 3 times with PBST then incubated for 1 hour (room temperature) with $2^{\circ} \mathrm{C}$ antibodies conjugated to a fluorophore. The blots were then washed 3 times with PBST, then 1 time with PBS to remove the excess Tween 20.

The bands were visualized using the Odyssey Classic Imaging System (Li-Cor Biosciences). The results were normalized to $\beta$-actin, GAPDH to account for discrepancies in loading and transfer. The loading controls were also evaluated for changes in expression. The loading control normalized values were then expressed as percent control values. The antibodies used were $\mathrm{GABA}_{\mathrm{A}}-\alpha 1$ (cat. no. AB5592-200; Millipore Corp., Billerica, MA), $\beta$-actin (cat. no. NB600-501; Novus Biologicals, Oakville, ON, Canada), HDAC1 (ab7028; Abcam, Cambridge, MA), HDAC2 (cat. no. ab7029; Abcam), and HDAC3 (cat. no. sc-11417; Santa Cruz Biotechnology, Dallas, TX).

\section{Chromatin Immunoprecipitation}

Chromatin immunoprecipitation (ChIP) assays were performed as previously described elsewhere (Kennedy et al., 2013) with some modifications. Neurons were rinsed with ice-cold PBS then crosslinked in $1 \%$ formaldehyde (Thermo Fisher Scientific, Waltham, MA) by agitation for 10 minutes at room temperature. The cross-linking reaction was quenched using $125 \mathrm{mM}$ glycine for 5 minutes at room temperature. The samples were centrifuged at $1600 \mathrm{~g}$ for 5 minutes at $4^{\circ} \mathrm{C}$ then washed with ice-cold PBS then spun again at $1600 \mathrm{~g}$ for 5 minutes at $4^{\circ} \mathrm{C}$.

Samples were lysed using ChIP lysis buffer (10 mM Tris-HCl, $\mathrm{pH}$ $8.0 ; 10 \mathrm{mM} \mathrm{NaCl} ; 0.2 \% \mathrm{v} / \mathrm{v} \mathrm{NP}-40$; and $1 \mathrm{mM}$ phenylmethylsulfonyl fluoride) on ice for 30 minutes and then homogenized and spun down at $2400 \mathrm{~g}$ for 10 minutes at $4^{\circ} \mathrm{C}$. Nuclei were lysed in nuclear lysis buffer (10 mM Tris-HCl, $\mathrm{pH}$ 8.0; $50 \mathrm{mM}$ EDTA; 1\%(v/v) SDS; and protease inhibitors) on ice for 10 minutes then sonicated to shear chromatin. Chromatin shearing of less than 500 bp was verified by running aliquots on $2 \%$ agarose gels.

After sonication, chromatin samples were spun for $18,000 \mathrm{~g}$ for 10 minutes at $4^{\circ} \mathrm{C}$, and the DNA concentrations were determined using a Nanodrop (Fisher Scientific). Equal amounts of chromatin were incubated overnight at $4^{\circ} \mathrm{C}$ with antibody, and an aliquot was set aside for input to ensure equal loading. PureProteome Protein G Magnetic beads (Millipore) were added to chromatin samples for 1 hour at $4^{\circ} \mathrm{C}$, then the complexes were washed, eluted in $1 \%(\mathrm{w} / \mathrm{v})$ SDS and $0.75 \%(\mathrm{w} / \mathrm{v})$ sodium bicarbonate buffer, and crosslinks were reversed overnight at $65^{\circ} \mathrm{C}$. DNA was purified using QIAquick PCR Purification Kit (Qiagen, Valencia, CA) and analyzed using SYBR Green Real-Time PCR Master Mixes (Thermo Scientific) following the manufacturer's instructions.

The primers used were Gabra1 Prom forward, 5'-CCCCCAAAATAGAGGAATGC-3'; and Gabra1 Prom reverse, 5'-AATAGGCGGTGACTTCATGC-3' . The antibodies used were Anti-Acetyl-Histone H3 (pan) (cat. no. 06-599; Millipore) and H3K9me3, (cat. no. ab8898; Abcam).

\section{Lentiviral Short Hairpin RNA knockdown}

Lentiviral experiments for HDAC1 knockdown experiments used short hairpin RNA (shRNA) from the RNAi Consortium library (Broad Institute, Cambridge, MA), provided by the UNC Lentiviral Core.

Target sequences for HDAC1 shRNAs were as follows:

5'-GCTTGGGTAATAGCAGCCATT-3'

5'-CCGGTATTTGATGGCTTGTTT-3'

5'-CCCTACAATGACTACTTTGAA-3'

5'-GCCAGTCATGTCCAAAGTAAT-3'

5'-GCGTTCTATTCGCCCAGATAA-3'
Lentivirus shRNA experiments for HDAC2 used shRNA plasmids from Origene (cat. no. TL7118660; Rockville, MD).

Target sequences for HDAC2 shRNAs were as follows:

5'-AGAAAGTGTGCTACTATTATGACGGTGAT-3'

5'-GCTTGTGATGAAGAGTTCTCAGATTCTGA-3'

5'-ACAACAGATCGCGTGATGACCGTCTCATT-3'

5' TCAAAGGTCACGCTAAATGTGTAGAAGTA-3'

The control experiments used a virus containing a scrambled DNA (SHC002, 5' -CCGGCGTGATCTTCACCGACAAGATCTCGAGATCTTGTCGGTGAAGATCACGTTTTT-3'; Sigma-Aldrich, St. Louis, MO). The shRNA plasmids were transformed in DH5 $\alpha$ cells, grown overnight at $37^{\circ} \mathrm{C}$, then purified using Maxiprep kits (Qiagen). DNA purity was checked with Nanodrop and rejected if $260 / 280$ or 230/260 values were below 1.8. Plasmids were then sequenced by the University of North Carolina (UNC) genome sequencing facility. Lentivirus was packaged by the UNC Lentiviral Core then aliquoted before use. Neurons were seeded on plates for 24 hours before virus was added directly to the medium (DIV 1). Four to five shRNAs were pooled to achieve maximum knockdown. On DIV 18, $\mathrm{H}_{2} \mathrm{O}$ or $\mathrm{EtOH}(50 \mathrm{mM})$ was added directly to the medium for 4 hours then the cells were harvested for Western blot analysis.

\section{Small-Interfering RNA Transfections}

Small-interfering RNA (siRNA) transfections were performed as we have previously described with some modifications (Werner et al., 2011). Neurons were grown until DIV 17 then transfected with 25 pmol Silencer HDAC3 siRNA (cat. no. 4390771; Thermo Fisher Scientific) or Silencer Select Negative Control No. 1 siRNA (cat. no. 4390843; Thermo Fisher Scientific) using Lipofectamine RNAiMax Reagent (Thermo Fisher Scientific) following the manufacturer's instructions. After 20 hours, the cells were exposed to $50 \mathrm{mM} \mathrm{EtOH}$ or $\mathrm{H}_{2} \mathrm{O}$ for 4 hours, then harvested for Western blot analysis.

\section{Small-Guide RNA Production}

Small-guide RNAs (sgRNAs) were designed in silico (crispr.mit.edu) based on experimentally determined algorithms described previously by Hsu et al. (2013) to be targeted at the promoter region or exon 5 and areas measured by ChIP primers. BsmBI sites (forward, 5'-TCCC-3'; reverse, $5^{\prime}-\mathrm{AAAC}-3^{\prime}$ ) were added to the CRISPR design to facilitate subcloning into the inducible vector (FgH1tUTG was a gift from Marco Herold [Addgene plasmid 70183]) (Aubrey et al., 2015). Oligos were annealed in a thermocycler starting at $95^{\circ} \mathrm{C}$ and then decreasing by $5^{\circ} \mathrm{C}$ every minute until $20^{\circ} \mathrm{C}$. Oligos $(100 \mu \mathrm{M}$ of both forward and reverse) were then phosphorylated with T4 PNK ligase according to manufacturer's instructions (cat. no. M0201S; New England Biolabs, Ipswich, MA).

Golden gate cloning was used to insert sgRNA oligos into FgH1tUTG (100 ng) by digesting with BsmbI (cat. no. ER0451; Fermentas, Vilnius, Lithuania) and annealing with $\mathrm{T} 7$ ligase (cat. no. M0318S; New England Biolabs) in the thermocycler: $37^{\circ} \mathrm{C}$ for 5 minutes then $23^{\circ} \mathrm{C}$ for 5 minutes, 15 cycles, and hold at $4^{\circ} \mathrm{C}$. The reaction was then transformed into homemade Sbtl3 cells by following manufacturer's instructions (cat. no. T3001; Zymo Research, Irvine, CA, made from cat. no. C737303; Thermo Scientific), plated on LB-Amp plates at $37^{\circ} \mathrm{C}$, and grown overnight. Individual colonies were selected and grown overnight in LB-Amp medium at $37^{\circ} \mathrm{C}$. Plasmid DNA was extracted using Miniprep kits (cat. no. A1340; Promega, Madison, WI) then sequenced using the H1 primer. For sgRNA sequences see Table 1 . Correctly sequenced clones were then packaged into lentivirus by the UNC Lentiviral Core.

\section{Subcloning dCas9-P300 into the Lentiviral Expression Vector}

We used a previously characterized dCas9-P300 construct (Hilton et al., 2015) for experiments. The dCas9-P300 construct was in a vector 
driven by the CMV promoter, which has poor expression in neurons, so we subcloned the dCas9-P300 constructs (Addgene 61357 and 61358, a generous gift from Dr. Charles Gersbach) into a lentiviral expression vector with an RFP promoter (Addgene 17619). We amplified dCas9-P300 out of the vector using primers designed with 20 bp overhangs for a Gibson assembly using PCR, then we extracted using PCR cleanup kits.

The lentiviral vector was cut using EcoRV (cat. no. R0195S; New England Biolabs), and the resultant fragments were purified using Qiagen Gel Extract kit (cat. no. 28704; Qiagen). The two fragments were incorporated using the Gibson assembly following the manufacturer's instructions (cat. no. E2611S; New England Biolabs). The clones were sequenced by Eton Bioscience (San Diego, CA) then packaged into lentivirus by the UNC Lentiviral Core. The following were the primers for cloning: forward, $5^{\prime}$ - GCTGGCTAGGTAAGCTTGATATGGACTACAAAGACCATGA-3'; $\alpha \nu \delta$ reverse, $5^{\prime}$-TAGGGCTGCAGGAATTCGATAGAAGCGTAGTCCGGAACGT-3' .

\section{Lentiviral Production of dCas9-VP64 and dCas9-KRAB}

Plasmids containing dCas9-VP64 (Addgene 53192) and dCas9KRAB (Addgene 71237) were a generous gift from Dr. Charles Gersbach. Of these, dCas9-VP64 was characterized by Kabadi et al. (2014), and dCas9-KRAB was characterized by Thakore et al. (2015). The plasmids were sequenced by Eton Biosciences then packaged into lentivirus by the UNC Lentiviral Core.

\section{Transduction of Cortical Neurons and Ethanol Exposure}

Equal volumes of three different lentiviruses with three different sgRNAs were mixed together with an equal volume of dCas9 vector (P300, P300 ${ }^{\mathrm{D} 1399 \mathrm{Y}}$, VP64, or KRAB) and transduced into neurons on DIV 15 with $10 \mu \mathrm{g}$ of polybrene (cat. no. H9268; Sigma Aldrich). On DIV 16, doxycycline in $\mathrm{H}_{2} \mathrm{O}$ was added to the cell culture medium (final concentration $=1 \mu \mathrm{g} / \mathrm{ml}$ ) to induce sgRNA expression (Aubrey et al., 2015). On DIV 18, $50 \mathrm{mM}$ EtOH or equivalent volume $\mathrm{ddH}_{2} \mathrm{O}$ was added for 4 hours then the cells were harvested for qPCR, ChIP, or Western blot analysis.

Statistics. All groups were randomly assigned. Two-way analysis of variance (ANOVA) was performed to determine statistical significance for all experiments. (ANOVA details for each experiment are given in the figure legends.) Bonferroni's post hoc test was used to perform multiple comparisons between groups to determine statistical significance. $P<0.05$ was considered statistically significant. Biologic replicates $(n)$ were performed by running the same experiment in different plates from a new cohort of animals.

\section{Results}

Histone Deacetylase Inhibitors Prevent Decreases in Gabra1 Expression Caused by Ethanol Exposure. We have previously shown that EtOH exposure $(50 \mathrm{mM}$ for 4 hours) in cultured cortical neurons causes a decrease in $\alpha 1$ expression in a crude membrane fraction (Kumar et al., 2010). We next evaluated whether $50 \mathrm{mM}$ EtOH exposure for 4 hours also caused a decrease in Gabra1 transcription (Fig. 1). Our results indicate that there is a robust decrease in Gabra1 expression similar to what is seen in vivo (Devaud et al., 1995b).

TABLE 1

sgRNA sequences

\begin{tabular}{lcc}
\hline \multicolumn{1}{c}{ ID } & \multicolumn{1}{c}{ Sequence 5' $\rightarrow 3^{\prime}$} & PAM \\
\hline Gabra1 Promoter \#1 & TAATACGTCCCAGCGCAAAC & CGG \\
Gabra1 Promoter \#2 & ATTTCACATCCGGTTTGCGC & TGG \\
Gabra1 Promoter \#3 & TTTCACATCCGGTTTGCGCT & GGG \\
Gabra1 Exon 5 \#1 & TGCCATCCTCTGTGATACGC & AGG \\
\hline
\end{tabular}

We next evaluated several different histone deacetylase inhibitors to determine whether this would prevent decreases in Gabra1 expression after 4 hours of $50 \mathrm{mM}$ EtOH exposure (Fig. 1). TSA (500 nM) coexposure prevented decreases in Gabra1 expression caused by EtOH exposure. Similarly, coexposure to SAHA ( $3 \mu \mathrm{M})$ also prevented decreases in Gabra1 expression.

Because both inhibitors are broad spectrum and inhibit all Zn-dependent histone deacetylases, we next used pharmacologic tools to evaluate whether certain isoforms were responsible. We used the HDAC3-selective inhibitor RGFP966 (Malvaez et al., 2013) at two different concentrations (24 and $80 \mathrm{nM}$ ) and found that coexposure of this compound prevented decreases in Gabra1 expression caused by EtOH exposure. In contrast, the class II selective inhibitor MC1568 failed to prevent EtOH-induced decreases in Gabra1 expression. Finally, the broad-spectrum HDAC inhibitor sodium butyrate did not prevent decreases in Gabra1 expression caused by EtOH exposure.

Knockdown of Class I HDACs Prevents Decreases in $\alpha 1$ Expression Caused by Ethanol Exposure. Because MC1568 exposure failed to prevent EtOH-induced decreases in Gabra1 expression, we hypothesized that inhibition of class I HDACs (1-3) would prevent EtOH-induced decreases in Gabra1 expression. To test this hypothesis, we used a genetic strategy to target HDAC1-3 (Fig. 2, A and B).

Knockdown of HDAC1 via a lentiviral strategy produced a decrease in HDAC1 expression (Fig. 2C, 58.78\% $\pm 3.41 \%$ control). Western blot analysis of the P2 membrane fraction revealed that knockdown of HDAC1 prevented the decrease of $\alpha 1$ expression after EtOH exposure (Fig. 2D). We tested for specificity of the knockdown of HDAC1 versus HDAC2 and HDAC3, and the qPCR analysis revealed that knockdown of HDAC1 did not decrease Hdac2 (0.96 \pm 0.1 fold control) or Hdac3 (0.82 \pm 0.08-fold control) (data not shown). Knockdown of HDAC2 using the same lentiviral strategy caused a decrease in HDAC2 expression (Fig. 2E, 48.96\% $\pm 6.25 \%$ control) and also prevented the decrease of $\alpha 1$ expression after $\mathrm{EtOH}$ exposure (Fig. 2F).

We next tested for specificity of the knockdown of HDAC2. The qPCR analysis revealed that knockdown of HDAC2 did not decrease Hdac1 (1.26 \pm 0.14 fold control) or Hdac3 $(0.83 \pm$ 0.09-fold control) (data not shown). Long-term knockdown of HDAC3 using a lentiviral strategy caused unviable neuronal cultures, so we used a siRNA strategy. Knockdown of HDAC3 with siRNA transfection caused a decrease in HDAC3 expression (Fig. 2G, 64.16\% $\pm 3.21 \%$ control) and also prevented decreases in $\alpha 1$ expression caused by EtOH exposure (Fig. $2 \mathrm{H})$. Knockdown of HDAC3 did not cause any change in Hdac1 $(0.99 \pm 0.08$-fold control) or Hdac2 (1.18 \pm 0.11 -fold control).

Design of Inducible CRISPR dCas9-P300 System To Study Acetylation at the Gabra1 Promoter. Because HDACs catalyze the removal of acetylation from histones (Wang et al., 2009), we hypothesized that EtOH was causing a decrease in Gabra1 expression and $\alpha 1$ expression by decreasing acetylation at the Gabra1 promoter. To test this idea, we used a previously described CRISPR dCas9 that is fused with the P300 acetyltransferase and specific sgRNAs to increase acetylation the Gabra1 promoter (Fig. 3, A and B) (Hilton et al., 2015). The sgRNAs were designed to target the region of -50 to $+250 \mathrm{bp}$ because we found decreased histone acetylation associated with the Gabra1 promoter (data not 


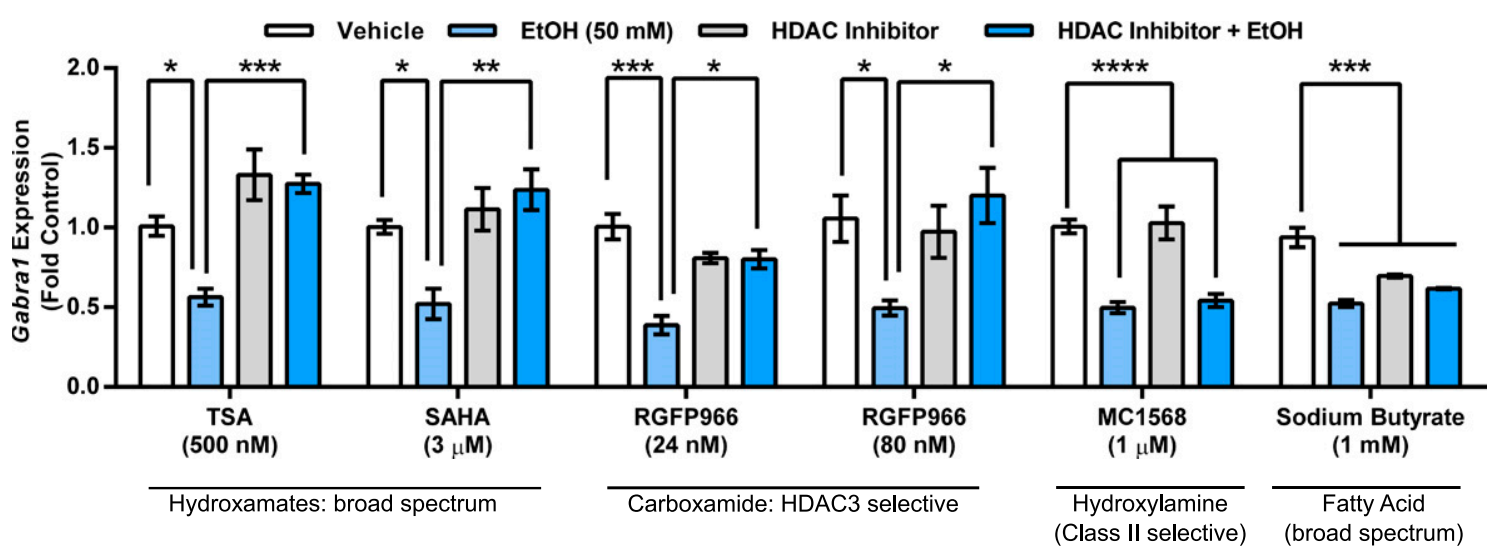

Fig. 1. Pharmacologic inhibition of class I HDACs prevents decrease in Gabra1 expression caused by EtOH exposure. Coexposure to TSA (500 nM) and EtOH (50 mM) prevents the EtOH-induced decrease in Gabra1 expression (two-way ANOVA interaction, $\mathrm{F}_{1,20}=25.68, P<0.0001, n=6$ ). Coexposure to SAHA $(3 \mu \mathrm{M})$ and EtOH $(50 \mathrm{mM})$ prevents decrease in Gabra1 expression (two-way ANOVA interaction, $\left.\mathrm{F}_{1,15}=7.831, P=0.0135, n=4-5\right)$. Coexposure to HDAC3-specific inhibitor and EtOH $(50 \mathrm{mM})$ at two concentrations of RGFP966 prevents decreases in Gabra1 expression: RGFP966 24 nM (two-way ANOVA interaction, $\mathrm{F}_{1,8}=26.47, P=0.0009, n=3$ ) and RGFP966 $80 \mathrm{nM}$ (two-way ANOVA interaction, $\mathrm{F}_{1,20}=7.758, P=0.0114, n=6$ ). Coexposure of class II HDAC inhibitor MC1568 $\left(1 \mu \mathrm{M}\right.$ ) with EtOH does not prevent decreases in Gabra1 expression (effect of EtOH, two-way ANOVA, $\mathrm{F}_{1,20}=64.49, P<$ $0.0001, n=6)$. Exposure to sodium butyrate $(1 \mathrm{mM}) \pm \mathrm{EtOH}(50 \mathrm{mM})$ causes a decrease in Gabra1 expression (two-way ANOVA interaction, $\mathrm{F}_{1,20}=17.16$, $P=0.0005, n=6$ ). Statistical significance is determined by two-way ANOVAs followed by Bonferroni post-hoc tests. $* P<0.05 ; * * P<0.01 ; * * * P<0.001$; $* * * * P<0.0001$. Each bar represents mean \pm S.E.M.

shown) and others have shown that this region has promoter activity ( $\mathrm{Hu}$ et al., 2008). We also designed an sgRNA targeting exon 5 because in vivo experiments demonstrated that $\mathrm{EtOH}$ did not change histone acetylation in this region, and this region is known to have low baseline acetylation (Wang et al., 2008).

Transduction of neurons on DIV 15 with both lentiviruses followed by doxycycline administration on DIV 16 produced lentiviral constructs colocalized to the same neurons (Fig. 3, C and D). Targeting of dCas9-P300 to the Gabra1 promoter caused a $349.8 \% \pm 64.3 \%$ increase in histone acetylation of the Gabra1 promoter region and prevented the EtOH-induced decrease in histone acetylation at the Gabra1 promoter (Fig. 4A, mock EtOH: $22.0 \% \pm 6.7 \%$ control; dCas9-P300 + EtOH: $412.4 \% \pm 117.5 \%$ control). Changes in histone acetylation correlated with a decrease in Gabra1 expression after EtOH (Fig. 4B, $0.22 \pm 0.02-$ fold control) and an increase in Gabra1 expression in the absence (Fig. 4B, $6.22 \pm 0.26$-fold control) and presence of EtOH (Fig. 4B, $6.60 \pm 1.04-$ fold control) with dCas9-P300 targeted to the Gabra1 promoter region. Western blot analysis of $\alpha 1$ expression revealed that dCas9-P300 targeted to the promoter region prevented the decrease of $\alpha 1$ expression caused by EtOH exposure (Fig. 4C).

We next used dCas9-P300(D1399Y), with no histone acetyltransferase activity, to determine whether histone acetyltransferase activity was necessary for preventing decreases caused by EtOH exposure. The qPCR analysis revealed that targeting dCas9-P300(D1399Y) to the Gabra1 promoter failed to prevent the decrease in Gabra1 expression caused by EtOH exposure (Fig. 4D). We then targeted dCas9-P300 to exon 5 of the Gabra1 gene to determine if acetylation of another region of the Gabra1 gene is sufficient to prevent decreases in Gabra1 expression after $\mathrm{EtOH}$ exposure. The qPCR analysis revealed that targeting dCas9-P300 to exon 5 of the Gabra1 gene failed to prevent decreases in Gabra1 expression caused by EtOH exposure (Fig. 4E).

We then tested whether targeting another dCas9 construct (dCas9-VP64) (Thakore et al., 2015) that is known to activate gene transcription via the Gabra1 promoter would prevent decreases in $\alpha 1$ expression caused by EtOH exposure. Western blot analysis revealed that dCas9-VP64 targeted at the Gabra 1 promoter was no different than mock transduced neurons in the presence of $\mathrm{EtOH}$ (Fig. 4F).

Targeted H3K9me3 at the Gabra1 Promoter Using dCas9-KRAB Fails To Prevent Decreases in Acetylation at the Gabra1 Promoter Caused by Ethanol Exposure. Histone post-translational modifications often work in conjunction or in opposition with one another to regulate gene transcription (Wang et al., 2008). Therefore, we examined whether changes in histone acetylation were due to interactions with another repressive histone post-translational modification. We used a dCas9 construct that is fused to the KRAB domain (Kabadi et al., 2014) to increase repressive H3K9me3 at the Gabra1 promoter (Fig. 5A).

ChIP analysis revealed that $\mathrm{EtOH}$ did not increase repressive H3K9me3 (Fig. 5B, 129.89\% $\pm 18.03 \%$ control). Targeting dCas9-KRAB to the Gabra1 promoter increased H3K9me3 associated with the Gabra1 promoter in both the absence (Fig. 5B, 393.76\% $\pm 21.71 \%$ control) and presence of EtOH (Fig. 5B, $432.05 \% \pm 132.84 \%$ control).

We next analyzed acetylation associated with the Gabra1 promoter after dCas-KRAB was targeted to the Gabra1 promoter in the presence or absence of EtOH. Only EtOH exposure caused a decrease of histone acetylation (Fig. 5C, mock EtOH $=41.44 \% \pm 4.26 \%$ control; dCas9-KRAB $+\mathrm{EtOH}$ $33.77 \% \pm 10.34 \%$ control). Western blot analysis suggested that both dCas9-KRAB transduction (dCas9-KRAB $+\mathrm{H}_{2} \mathrm{O}$ : $66.87 \% \pm 15.27 \%$ control) and EtOH exposure (no dCas9$\mathrm{KRAB}+\mathrm{EtOH} 71.28 \% \pm 4.25 \%$ control) caused a decrease in $\alpha 1$ expression (Fig. 5D), but there was no further decrease in $\alpha 1$ expression when both $\mathrm{dCas} 9-\mathrm{KRAB}$ and $\mathrm{EtOH}$ were present $(74.54 \% \pm 9.48 \%$ control). However, these changes failed to reach statistical significance when analyzed by two-way ANOVA.

\section{Discussion}

Ethanol exposure decreases $\alpha 1$ protein expression both in vitro and in vivo and is thought to underlie $\mathrm{GABA}_{\mathrm{A}}-\mathrm{R}$ 
A

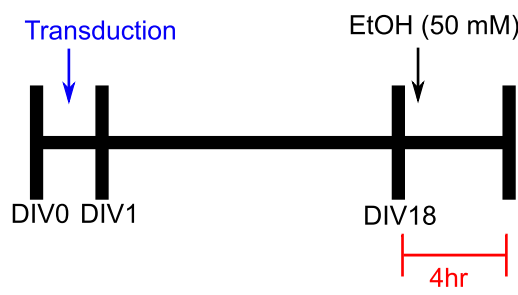

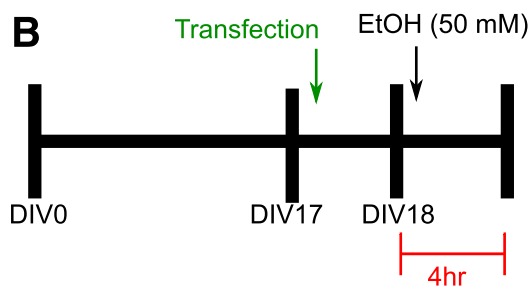

HDAC2

$\mathbf{E}$

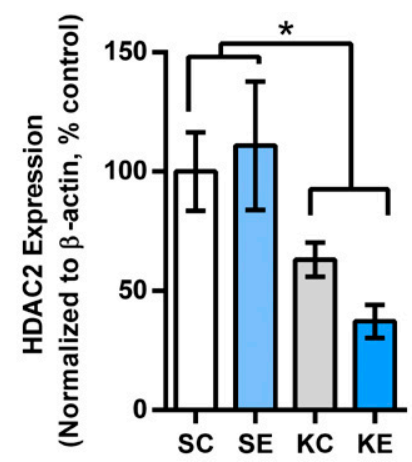

$\mathbf{F}$

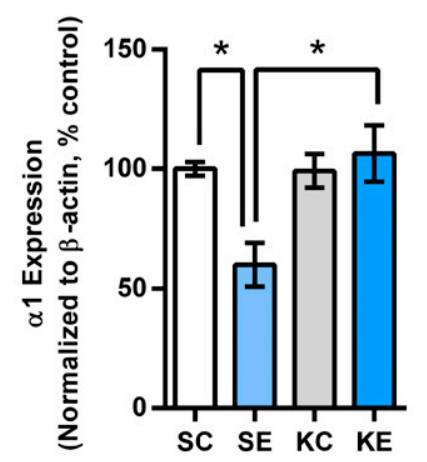

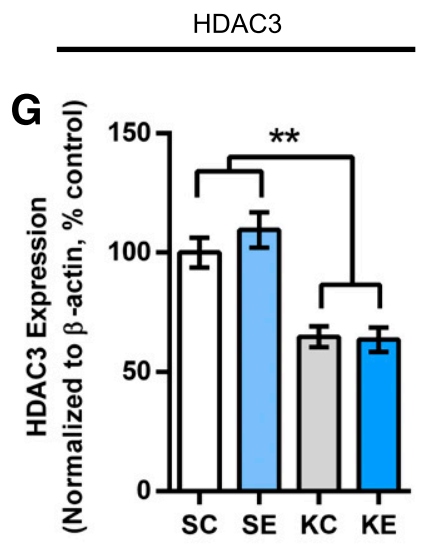

H

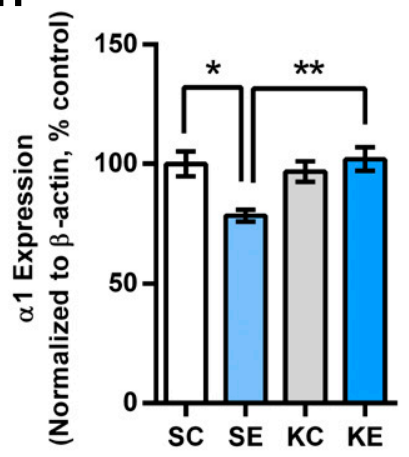

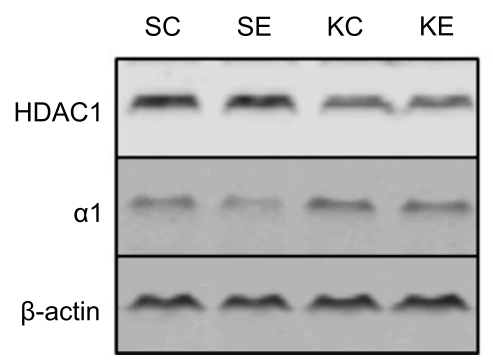
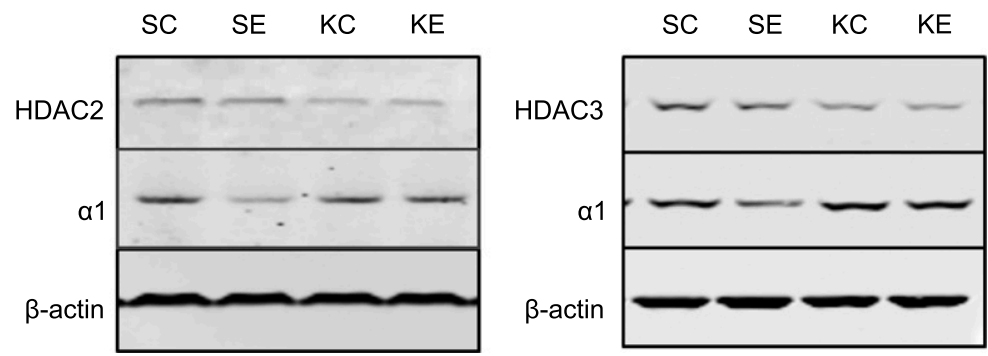

Fig. 2. Knockdown of HDAC1-3 prevents decreases in $\alpha 1$ expression caused by EtOH exposure. (A) Schematic demonstrating the experimental outline for knockdown of HDAC1 and HDAC2 using shRNA delivered via lentiviral transduction. (B) Schematic demonstration of the experimental outline for knockdown of HDAC3 using siRNA via transfection. (C) Quantification of HDAC1 expression after HDAC1 knockdown (effect of HDAC1 knockdown, two-way ANOVA, $\mathrm{F}_{1,20}=105.1, P<0.0001, n=6$ ). (D) Quantification of HDAC2 expression after HDAC2 knockdown (effect of HDAC2 knockdown, twoway ANOVA, $\mathrm{F}_{1,1}=10.24, P=0.0047, n=5-6$ ). (E) Quantification of HDAC3 expression after HDAC3 knockdown (effect of HDAC3 knockdown, two-way ANOVA, $\mathrm{F}_{1,20}=47.41, P<0.0001, n=6$ ). (F) Representative Western blots and quantification of $\alpha 1$ expression after HDAC1 knockdown (effect of HDAC1 knockdown, two-way ANOVA, $\mathrm{F}_{1,20}=20.84, P=0.0002 . n=6$ ). (G) Representative Western blots and quantification of $\alpha 1$ expression after HDAC2 knockdown (two-way ANOVA interaction, $\mathrm{F}_{1,17}=7.521, P=0.0139, n=4-6$ ). (H) Representative Western blots and quantification of $\alpha 1$ expression after HDAC3 knockdown (two-way ANOVA interaction, $\mathrm{F}_{1,19}=9.403, P=0.0064, n=5-6$ ). Each bar represents the mean \pm S.E.M. Statistical significance is determined by two-way ANOVA followed by Bonferroni post hoc tests. $* P<0.05$; $* * P<0.01$. KC, knockdown of $\mathrm{HDAC}+\mathrm{H}_{2} \mathrm{O}$ exposure; $\mathrm{KE}$, knockdown of HDAC + EtOH exposure; $\mathrm{SC}$, scrambled virus or siRNA $+\mathrm{H}_{2} \mathrm{O}$ exposure; $\mathrm{SE}$, scrambled virus or siRNA + EtOH exposure.

hypofunction and EtOH withdrawal symptoms in rodents (Devaud et al., 1995b, 1997; Liang et al., 2004, 2007; Kumar et al., 2009). Decreases in $\alpha 1$ protein expression has been shown to occur at the same time that there are decreases in Gabra1 gene expression (Devaud and Morrow, 1995; Devaud et al., 1997), suggesting that a transcriptional mechanism might be involved. Our results reveal that EtOH controls Gabra1 gene expression through a histone deacetylation mechanism that can be prevented by interventions on either the pharmacologic, genetic, or epigenetic level. Histone 
A

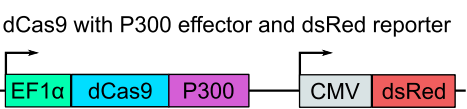

Inducible sgRNA construct with eGFP reporter \begin{tabular}{l}
$-\mathrm{H} 1$ \\
\hline
\end{tabular}
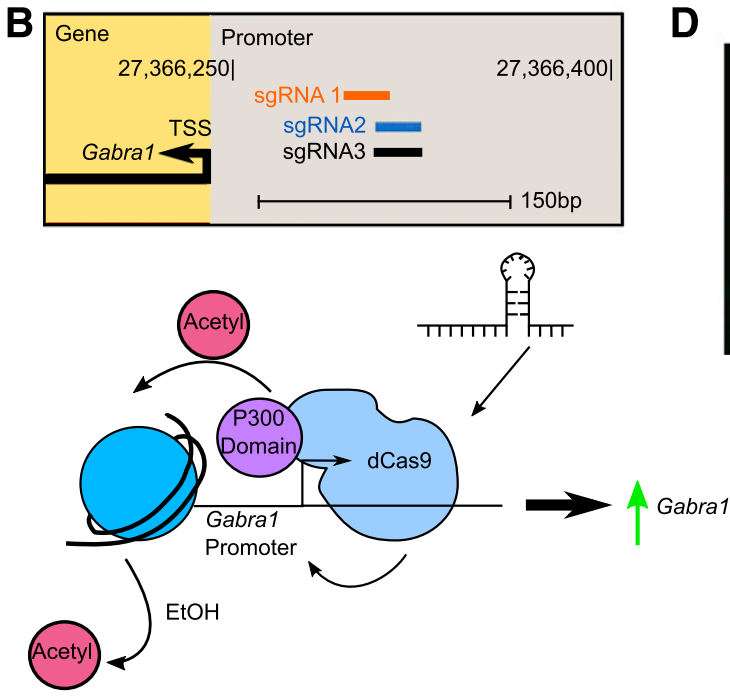

C

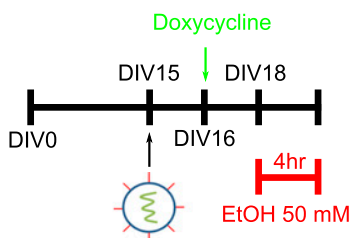

Lentiviral Transductions

D

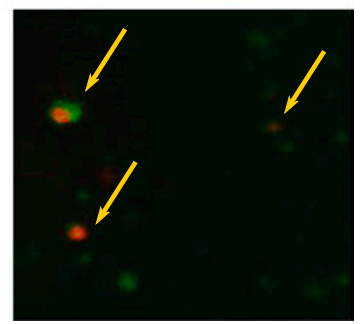

Fig. 3. Experimental strategy for dCas9-P300 use in probing histone acetylation associated with the Gabra1 promoter after EtOH exposure. (A) Plasmid maps demonstrating the vector for dCas9-P300 with the dsRed reporter and the inducible sgRNA constructs with eGFP reporters. (B) Experimental strategy to determine if preventing EtOH-induced histone deacetylation at the Gabra1 promoter prevents decreases in Gabra1 expression. (C) Schematic demonstrating experimental timeline for experiments. The dayCas9 constructs in lentiviral particles are transduced at DIV 15, sgRNA production is induced on DIV 16 by doxycycline, and $50 \mathrm{mM} \mathrm{EtOH}$ is added to neurons for 4 hours on DIV 18. (D) Representative epifluorescent image showing colocalization of dsRed and eGFP to demonstrate transduction of cortical neurons with dCas9-P300 and sgRNA, respectively. deacetylation associated with the Gabra1 promoter is likely driven by class I HDACs, as knockdown of HDAC1-3 prevented EtOH-induced decreases of $\alpha 1$ protein expression.

Previous studies have found that HDAC inhibitors prevent decreases in $\alpha 1$ protein expression after chronic EtOH exposure (Arora et al., 2013) or via the $\alpha 1$ (A322D) gene mutation that decreases $\alpha 1$ expression found in epilepsy (Di et al., 2013). However, neither of these studies determined whether transcriptional mechanisms via epigenetic modulation were involved.

Our results indicate that EtOH exposure causes a decrease in $\alpha 1$ protein expression through histone deacetylation associated with the Gabra1 promoter. Deacetylation of histone associated with promoters often correlates with decreases in gene transcription, although there are cases in which the gene silenced appears to preclude changes in histone acetylation (Wang et al., 2008).

Importantly, until recent advances in gene targeting technology, most studies have only examined correlations between gene transcription and histone acetylation. We used several strategies to determine that histone acetylation associated with the Gabra1 promoter was contributing to decreases in Gabra1 expression. First, EtOH exposure caused a decrease in histone acetylation associated with the Gabra1 promoter, and this was prevented by targeting histone acetylation to this location. Second, targeting a generic Cas9 activator (dCas9VP64) to this region did not prevent decreases in $\alpha 1$ expression caused by EtOH exposure. Third, increasing repressive $\mathrm{H} 3 \mathrm{~K} 9 \mathrm{me} 3$ at this region did not interfere with the EtOHinduced decrease in $\mathrm{H} 3$ acetylation.

Together, these results suggest that histone deacetylation associated with the Gabra1 promoter regulates the decrease in $\alpha 1$ expression in response to $\mathrm{EtOH}$, elucidating a new regulatory mechanism for EtOH's control of $\alpha 1$ expression.
This does not preclude the possibility that histone acetylation is also regulating trafficking and/or folding in response to EtOH and that HDAC inhibitors are working to prevent changes on that level. However, our results appear to suggest that a transcriptional mechanism is likely driving the changes, as targeted acetylation of the Gabra1 promoter prevents changes in $\mathrm{GABA}_{\mathrm{A}}-\mathrm{R} \alpha 1$ protein expression.

The region of the Gabra1 promoter where we found increased histone acetylation contains 797 putative transcription factor binding motifs (Mathelier et al., 2016). Of these transcription factors, cAMP response element binding (CREB) and CREB-binding protein sites were identified, which have previously been implicated in controlling Gabra1 transcript and surface expression in animal models of epilepsy (Hu et al., 2008; Grabenstatter et al., 2012). Deficits in CREB-binding protein have been implicated in models of alcohol dependence in the amygdala (Pandey et al., 2005, 2017), an area that shows evidence of $\mathrm{GABA}_{\mathrm{A}}$ receptor hypofunction (Herman et al., 2013, 2016). Thus, decreases in histone acetylation at the Gabra1 promoter may initiate signaling cascades that are involved in decreasing $\mathrm{GABA}_{\mathrm{A}}-\mathrm{R} \alpha 1$ expression and function. Future experiments should consider the transcription factors involved to specifically determine EtOH effects on Gabra1 gene transcription due to changes in histone acetylation.

Pharmacologic and knockdown experiments have suggested that class I HDACs are responsible for $\mathrm{EtOH}$-induced changes in Gabra1 expression. The class I HDACs include HDAC(1-3) and are typically localized to the nucleus (Haberland et al., 2009). HDAC1 and HDAC2 are nearly identical and are often found in the same repressive complexes (Haberland et al., 2009). However, HDAC2 has been suggested to be involved in the development of alcohol dependence in rodent models (Arora et al., 2013; Moonat et al., 2013; López-Moreno et al., 2015), and another study has shown that HDAC2 is 
A

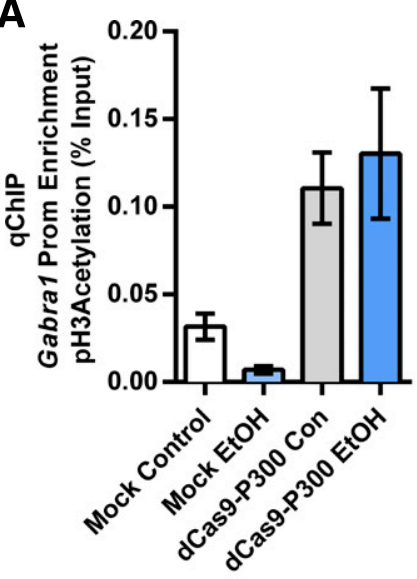

D

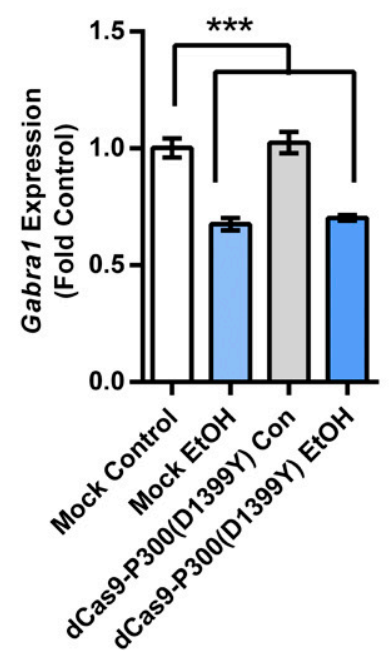

B

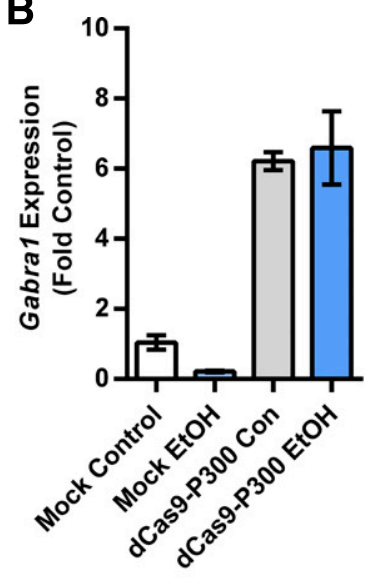

E

sgRNA targets Exon 5

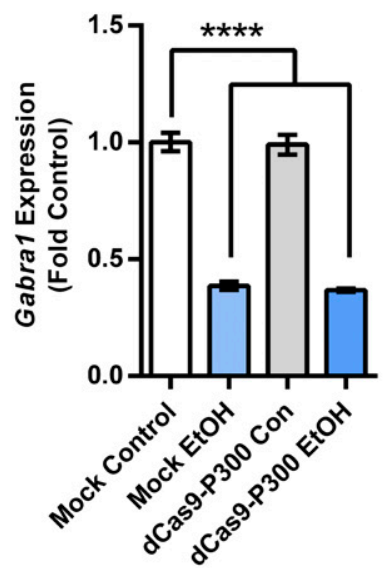

C
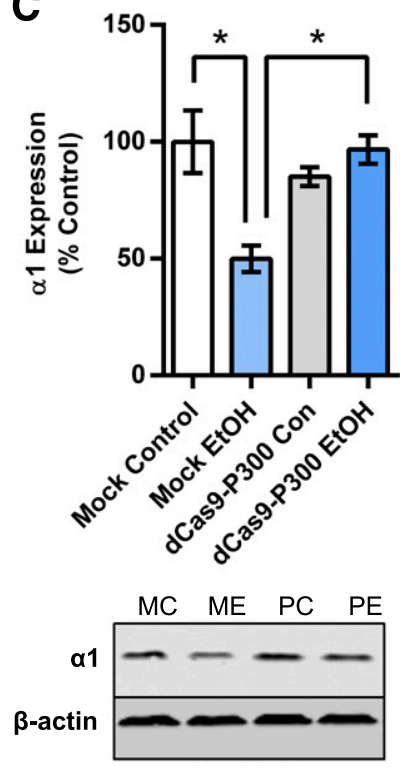

F

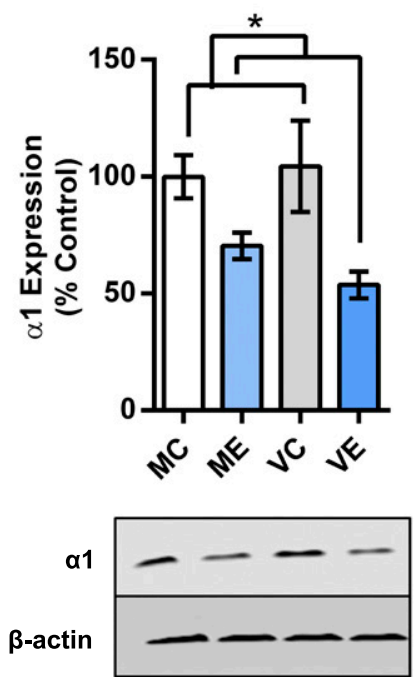

Fig. 4. dCas9-P300 prevents deacetylation of the Gabra1 promoter and decreases in Gabra1 expression caused by EtOH exposure. (A) Quantitative chromatin immunoprecipitation using a pan-acetyl $\mathrm{H} 3$ antibody reveals that targeting the Gabra1 promoter with dCas9-P300 increases acetylation at the Gabra1 promoter (effect of dCas9-P300, two-way ANOVA, $\mathrm{F}_{1,8}=22.10, P=0.0015$ ). (B) Quantitative PCR analysis reveals that targeting the Gabra1 promoter with dCas9-P300 increases Gabra1 expression (effect of dCas9-P300, two-way ANOVA, $\mathrm{F}_{1,8}=18.81, P=0.0025$ ). Asterisks shown are for twoway ANOVA significance. (C) Western blot analysis reveals that targeting the Gabra1 promoter with dCas9-P300 prevents the decrease in GABA $\mathrm{A}$-R $\alpha 1$ expression (two-way ANOVA, $\mathrm{F}_{1,8}=14.32, P=0.0054$ ). (D) Targeting the Gabra1 promoter with a mutant dCas9-P300 that lacks histone acetyltransferase activity fails to prevent the decrease in Gabra1 expression caused by EtOH (two-way ANOVA, ANOVA $\mathrm{F}_{1,8}=91.08, P<0.0001$ ). $(\mathrm{E})$ Targeting Gabra1 exon 5 with dCas9-P300 fails to prevent decreases in Gabra1 expression caused by EtOH exposure (two-way ANOVA, $\mathrm{F}_{1,8}=415.0, P<$ 0.0001). (F) Generic activation with a VP64 domain fails to prevent decreases in $\alpha 1$ expression caused by EtOH exposure (significant effect of EtOH, twoway ANOVA $F_{18}=12.16, P=0.0082$ ). Each bar represents mean \pm S.E.M.; $n=3$ independent experiments. Statistical significance is determined by twoway ANOVA followed by Bonferroni post hoc tests. ${ }^{*} P<0.05$; $* * * P<0.001$; $* * * * P<0.0001$.

up-regulated by EtOH at 4 hours in a neuronal cell line and that this effect can be blocked by TSA (Agudelo et al., 2011). However, none of the previous studies reported evaluating HDAC1 or HDAC3 expression.

Interestingly, HDAC2 knockdown in the hippocampus causes a decrease in excitatory transmission and increase in inhibitory transmission but has no effect on $\alpha 1$ expression (Hanson et al., 2013). Other experiments have shown that HDAC2 but not HDAC1 overexpression regulates memory formation and synaptic plasticity (Guan et al., 2009), while another study has suggested that HDAC1 but not HDAC2 regulates locomotor effects of cocaine (Kennedy et al., 2013). Finally, MS-275 [ $N$-(2-aminophenyl)-4-[ $N$-(pyridin-3-yl-methoxycarbonyl) aminomethyl]benzamide], which inhibits class I HDACs, prevented cocaine-induced increases in Gabra1 expression (Kennedy et al., 2013).

Interestingly, HDAC knockdown did not cause compensatory changes in other HDAC isoform expressions that we 
A

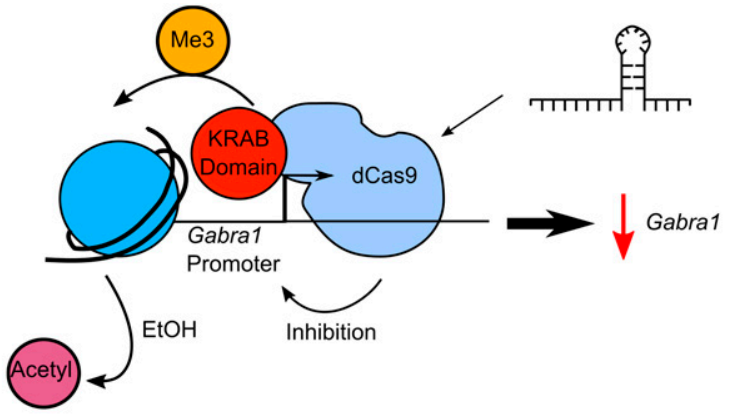

B

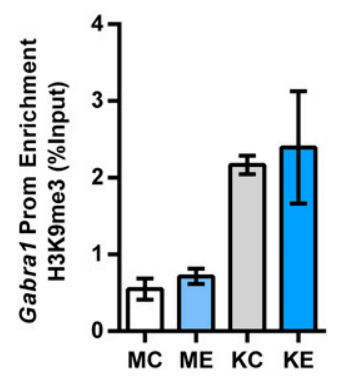

C

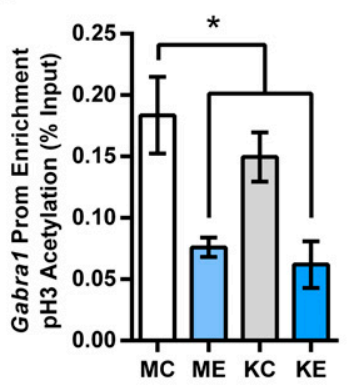

D

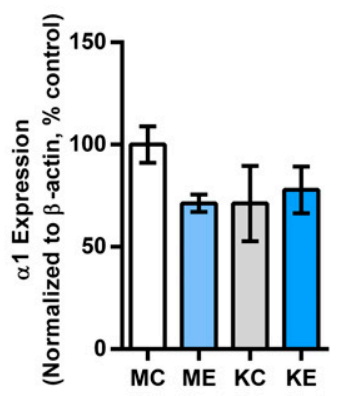

Fig. 5. Increasing repressive $\mathrm{H} 3 \mathrm{~K} 9 \mathrm{me} 3$ does not alter decreases in Gabra1 expression caused by deacetylation. (A) Schematic of the experimental strategy to use dCas9-KRAB targeted at the Gabra1 promoter to increase H3K9me3. (B) ChIP reveals that targeting dCas9-KRAB to the Gabra1 promoter increases H3K9me3 (effect of dCas9KRAB, two-way ANOVA, $\mathrm{F}_{1,8}=18.81, P=0.0025$ ). (C) ChIP reveals that $\mathrm{H} 3$ acetylation at the Gabra1 promoter is only decreased in the presence of EtOH (effect of EtOH, two-way ANOVA, $\mathrm{F}_{1,8}=21.05, P=$ 0.0018 ). (D) Western blot analysis reveals that both dCas9-KRAB and EtOH trend toward decreased $\alpha 1$ expression, but dCas9-KRAB does not alter the effect of EtOH (interaction between dCas9-KRAB and EtOH, two-way ANOVA, $\mathrm{F}_{1,8}=2.21, P=0.18$ ). Each bar represents mean \pm S.E.M. of three independent experiments. Statistical significance is determined by two-way ANOVA followed by Bonferroni post hoc test. ${ }^{*} P<0.05$. KC, dCas9KRAB transduction $\pm \mathrm{H}_{2} \mathrm{O}$; KE, dCas9-KRAB transduction + EtOH; MC, mock transduction \pm $\mathrm{H}_{2} \mathrm{O} ; \mathrm{ME}$, mock transduction + EtOH.

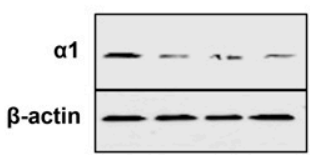

measured via $\mathrm{qPCR}$, and this effect has been previously reported (Kennedy et al., 2013; Kuzmochka et al., 2014). However, this does not preclude compensatory changes that could occur or that we may not have observed due to the temporal and spatial constraints of our study.

Neurons were cultured from mixed sex (50\% female/male) rat cerebral cortex. Thus, it is noteworthy that EtOH induced the same changes in Gabra1 and $\mathrm{GABA}_{\mathrm{A}}-\mathrm{R} \alpha 1$ subunit expression in these neurons as previously reported in various in vivo studies in male rat cortex after chronic EtOH exposure that produced EtOH dependence (Montpied et al., 1991; Mhatre and Ticku, 1992; Devaud et al., 1995b, 1997; Cagetti et al., 2003; Sanna et al., 2003; Liang et al., 2004), but distinct from one study in female rats (Devaud et al., 1999) where no change in $\mathrm{GABA}_{\mathrm{A}}-\mathrm{R} \alpha 1$ subunit expression was observed. This comparison raises the possibility that EtOH effects on histone deacetylation may also involve sex differences that should be explored in future studies.

Histone deacetylase inhibitors have been suggested as a promising treatment of alcohol use disorders (Pandey et al., 2008; Arora et al., 2013; Warnault et al., 2013; Simon-O'Brien et al., 2015). The present results further support the use of class I histone deacetylase inhibitors for the treatment of alcohol use disorders. GABA $\mathrm{A}_{\mathrm{A}}-\mathrm{R}$ have long been known to be down-regulated after chronic EtOH use in both humans (Lingford-Hughes et al., 1997, 1998, 2000; Taylor et al., 2008) and rodents (Morrow et al., 1991; Liang et al., 2007), and this downregulation causes benzodiazepine cross-tolerance (LingfordHughes et al., 1997, 1998, 2000; Cagetti et al., 2003; Liang et al., 2007; Taylor et al., 2008). Interventions that effect $\mathrm{GABA}_{\mathrm{A}}-\mathrm{R}$ expression on a transcriptional level may bypass problems associated with using benzodiazepines in acute alcohol withdrawal syndrome, as benzodiazepines have high abuse potential, are potentially lethal when combined with alcohol, and show cross-tolerance with EtOH (Hollister, 1990). Conversely, HDAC inhibitors have been shown to decrease EtOH and cocaine self-administration (Romieu et al., 2008; Simon-O'Brien et al., 2015), making them less likely to be abused.

Additionally, human alcohol use disorder patients exhibit changes in a number of different genes (Farris et al., 2015) that may be related to changes in the epigenome (Ponomarev et al., 2012). Our results indicate that careful selection of the proper pharmacologic inhibitors for preventing epigenetic changes in alcohol use disorders is required, as sodium butyrate failed to prevent changes in Gabra1 expression, although this inhibitor has been shown to prevent drinking in vivo (Simon-O'Brien et al., 2015). Sodium butyrate is a GABA analog and may have agonist activity at receptors, leading to activity-dependent down-regulation of these receptors. Thus, interventions for alcohol dependence may require targeting molecular components that control acetylation mechanisms rather than individual genes, as demonstrated in the current study using dCas9-P300. However, this system has useful experimental value because it allows for determination of specific epigenetic mechanisms associated with specific genes.

In conclusion, we present a new signaling pathway activated by chronic EtOH exposure and withdrawal that involves histone deacetylation associated with the Gabra1 promoter, which drives decreases in Gabra1 transcription in cortical cultured neurons. Finally, we suggest that histone deacetylase inhibitors may be a useful therapeutic intervention for the treatment of alcohol use disorders and possibly other diseases where $\alpha 1$ expression is dysregulated. 


\section{Authorship Contributions}

Participated in research design: Bohnsack, Morrow.

Conducted experiments: Bohnsack, Patel.

Performed data analysis: Bohnsack, Patel, Morrow.

Wrote or contributed to writing of the manuscript: Bohnsack,

\section{Morrow.}

\section{References}

Agudelo M, Gandhi N, Saiyed Z, Pichili V, Thangavel S, Khatavkar P, Yndart-Arias A, and Nair M (2011) Effects of alcohol on histone deacetylase 2 (HDAC2) and the neuroprotective role of trichostatin A (TSA). Alcohol Clin Exp Res 35:1550-1556.

Amato L, Minozzi S, Vecchi S, and Davoli M (2010) Benzodiazepines for alcohol withdrawal. Cochrane Database Syst Rev 3:CD005063.

Arora DS, Nimitvilai S, Teppen TL, McElvain MA, Sakharkar AJ, You C, Pandey SC, and Brodie MS (2013) Hyposensitivity to gamma-aminobutyric acid in the ventral tegmental area during alcohol withdrawal: reversal by histone deacetylase inhibitors. Neuropsychopharmacology 38:1674-1684.

Aubrey BJ, Kelly GL, Kueh AJ, Brennan MS, O’Connor L, Milla L, Wilcox S, Tai L, Strasser A, and Herold MJ (2015) An inducible lentiviral guide RNA platform enables the identification of tumor-essential genes and tumor-promoting mutations in vivo. Cell Rep 10:1422-1432.

Bohnsack JP, Carlson SL, and Morrow AL (2016) Differential regulation of synaptic and extrasynaptic $\alpha 4 \mathrm{GABA}_{\mathrm{A}}$ ) receptor populations by protein kinase $\mathrm{A}$ and protein kinase C in cultured cortical neurons. Neuropharmacology 105:124-132.

Broide RS, Redwine JM, Aftahi N, Young W, Bloom FE, and Winrow CJ (2007) Distribution of histone deacetylases $1-11$ in the rat brain. J Mol Neurosci 31 47-58.

Cagetti E, Liang J, Spigelman I, and Olsen RW (2003) Withdrawal from chronic intermittent ethanol treatment changes subunit composition, reduces synaptic function, and decreases behavioral responses to positive allosteric modulators of $\mathrm{GABA}_{\mathrm{A}}$ receptors. Mol Pharmacol 63:53-64.

Cagetti E, Pinna G, Guidotti A, Baicy K, and Olsen RW (2004) Chronic intermittent ethanol (CIE) administration in rats decreases levels of neurosteroids in hippocampus, accompanied by altered behavioral responses to neurosteroids and memory function. Neuropharmacology 46:570-579.

Carlson SL, Kumar S, Werner DF, Comerford CE, and Morrow AL (2013) Ethanol activation of protein kinase A regulates GABAA $\alpha 1$ receptor function and traf ficking in cultured cerebral cortical neurons. J Pharmacol Exp Ther 345:317-325.

Devaud LL and Morrow AL (1995) Interactions between neuroactive steroids and ethanol at GABAA receptors: effects of ethanol withdrawal, in Stress, Gender and Alcohol-Seeking Behavior, NIAAA Research Monograph No 29 (Hunt WA and Zakhari S eds) pp 219-240, U.S. Gov. Printing Office, Washington, DC.

Devaud LL, Purdy RH, and Morrow AL (1995a) The neurosteroid, $3 \alpha$-hydroxy-5 $\alpha$-pregnan-20-one, protects against bicuculline-induced seizures during ethanol withdrawal in rats. Alcohol Clin Exp Res 19:350-355.

Devaud LL, Smith FD, Grayson DR, and Morrow AL (1995b) Chronic ethanol consumption differentially alters the expression of $\gamma$-aminobutyric acidA receptor subunit mRNAs in rat cerebral cortex: competitive, quantitative reverse transcriptase-polymerase chain reaction analysis. Mol Pharmacol 48:861-868.

Devaud LL, Fritschy J-M, Sieghart W, and Morrow AL (1997) Bidirectional alterations of $\left.\mathrm{GABA}_{(}\right)$receptor subunit peptide levels in rat cortex during chronic ethanol consumption and withdrawal. J Neurochem 69:126-130.

Devaud LL, Matthews DB, and Morrow AL (1999) Gender impacts behavioral and neurochemical adaptations in ethanol-dependent rats. Pharmacol Biochem Behav 64:841-849.

Di X-J, Han D-Y, Wang Y-J, Chance MR, and Mu T-W (2013) SAHA enhances proteostasis of epilepsy-associated $\alpha 1(\mathrm{~A} 322 \mathrm{D}) \beta 2 \gamma 2 \mathrm{GABA}(\mathrm{A})$ receptors. Chem Biol $\mathbf{2 0}$ $1456-1468$

Doudna JA and Charpentier E (2014) Genome editing. The new frontier of genome engineering with CRISPR-Cas9. Science 346:1258096.

Farris SP, Arasappan D, Hunicke-Smith S, Harris RA, and Mayfield RD (2015) Transcriptome organization for chronic alcohol abuse in human brain. Mol Psy chiatry 20:1438-1447.

Fatemi SH, Reutiman TJ, Folsom TD, and Thuras PD (2009) $\left.\operatorname{GABA}_{\mathrm{A}}\right)$ receptor downregulation in brains of subjects with autism. $J$ Autism Dev Disord 39 $223-230$.

Grabenstatter HL, Russek SJ, and Brooks-Kayal AR (2012) Molecular pathways controlling inhibitory receptor expression. Epilepsia 53 (Suppl 9):71-78.

Guan J-S, Haggarty SJ, Giacometti E, Dannenberg J-H, Joseph N, Gao J, Nieland TJF, Zhou Y, Wang X, Mazitschek R, et al. (2009) HDAC2 negatively regulates memory formation and synaptic plasticity. Nature 459:55-60.

Haberland M, Montgomery RL, and Olson EN (2009) The many roles of histone deacetylases in development and physiology: implications for disease and therapy. Nat Rev Genet 10:32-42.

Hanson JE, Deng L, Hackos DH, Lo SC, Lauffer BE, Steiner P, and Zhou Q (2013) Histone deacetylase 2 cell autonomously suppresses excitatory and enhances inhibitory synaptic function in CA1 pyramidal neurons. $J$ Neurosci 33:5924-5929.

Herman MA, Contet C, Justice NJ, Vale W, and Roberto M (2013) Novel subunitspecific tonic GABA currents and differential effects of ethanol in the central amygdala of CRF receptor-1 reporter mice. J Neurosci 33:3284-3298.

Herman MA, Contet C, and Roberto M (2016) A functional switch in tonic gaba currents alters the output of central amygdala corticotropin releasing factor receptor-1 neurons following chronic ethanol exposure. $J$ Neurosci $\mathbf{3 6}$ 10729-10741.

Hilton IB, D'Ippolito AM, Vockley CM, Thakore PI, Crawford GE, Reddy TE, and Gersbach CA (2015) Epigenome editing by a CRISPR-Cas9-based acetyltransferase activates genes from promoters and enhancers. Nat Biotechnol 33: 510-517.
Hoftman GD, Volk DW, Bazmi HH, Li S, Sampson AR, and Lewis DA (2015) Altered cortical expression of GABA-related genes in schizophrenia: illness progression vs developmental disturbance. Schizophr Bull 41:180-191.

Hollister LE (1990) Interactions between alcohol and benzodiazepines. Recent Dev Alcohol 8:233-239.

Hsu PD, Scott DA, Weinstein JA, Ran FA, Konermann S, Agarwala V, Li Y, Fine EJ, Wu X, Shalem O, et al. (2013) DNA targeting specificity of RNA-guided Cas9 nucleases. Nat Biotechnol 31:827-832.

Hu Y, Lund IV, Gravielle MC, Farb DH, Brooks-Kayal AR, and Russek SJ (2008) Surface expression of $\mathrm{GABA}_{\mathrm{A}}$ receptors is transcriptionally controlled by the interplay of cAMP-response element-binding protein and its binding partner inducible cAMP early repressor. J Biol Chem 283:9328-9340.

Kabadi AM, Ousterout DG, Hilton IB, and Gersbach CA (2014) Multiplex CRISPR/Cas9-based genome engineering from a single lentiviral vector. Nucleic Acids Res 42: 147.

Kennedy PJ, Feng J, Robison AJ, Maze I, Badimon A, Mouzon E, Chaudhury D, Damez-Werno DM, Haggarty SJ, Han MH, et al. (2013) Class I HDAC inhibition blocks cocaine-induced plasticity by targeted changes in histone methylation. Nat Neurosci 16:434-440.

Kralic JE, Criswell HE, Osterman JL, O’Buckley TK, Wilkie ME, Matthews DB Hamre K, Breese GR, Homanics GE, and Morrow AL (2005) Genetic essential tremor in gamma-aminobutyric acid $_{\mathrm{A}}$ receptor $\alpha 1$ subunit knockout mice. J Clin Invest 115:774-779.

Kumar S, Porcu P, Werner DF, Matthews DB, Diaz-Granados JL, Helfand RS, and Morrow AL (2009) The role of $\left.\operatorname{GABA}_{\mathrm{A}}\right)$ receptors in the acute and chronic effects of ethanol: a decade of progress. Psychopharmacology (Berl) 205:529-564. Kumar S, Sieghart W, and Morrow AL (2002) Association of protein kinase C with GABA(A) receptors containing $\alpha 1$ and $\alpha 4$ subunits in the cerebral cortex: selective effects of chronic ethanol consumption. J Neurochem 82:110-117.

Kumar S, Suryanarayanan A, Boyd KN, Comerford CE, Lai MA, Ren Q, and Morrow $\mathrm{AL}$ (2010) Ethanol reduces $\mathrm{GABA}_{\mathrm{A}} \alpha 1$ subunit receptor surface expression by a protein kinase $\mathrm{C} \gamma$-dependent mechanism in cultured cerebral cortical neurons. $\mathrm{Mol}$ Pharmacol 77:793-803.

Kuzmochka C, Abdou H-S, Haché RJG, and Atlas E (2014) Inactivation of histone deacetylase 1 (HDAC1) but not HDAC2 is required for the glucocorticoiddependent CCAAT/enhancer-binding protein $\alpha(\mathrm{C} / \mathrm{EBP} \alpha)$ expression and preadipocyte differentiation. Endocrinology 155:4762-4773.

Liang J, Cagetti E, Olsen RW, and Spigelman I (2004) Altered pharmacology of synaptic and extrasynaptic $\mathrm{GABA}_{\mathrm{A}}$ receptors on CA1 hippocampal neurons is consistent with subunit changes in a model of alcohol withdrawal and dependence. $J$ Pharmacol Exp Ther 310:1234-1245.

Liang J, Suryanarayanan A, Abriam A, Snyder B, Olsen RW, and Spigelman I (2007) Mechanisms of reversible $\mathrm{GABA}_{\mathrm{A}}$ receptor plasticity after ethanol intoxication. $J$ Neurosci 27:12367-12377.

Lingford-Hughes AR, Acton PD, Gacinovic S, Busatto GF, Costa DC, Boddington S, Marshall JE, Ell PJ, Kerwin RW, and Pilowsky LS (1997) Reduced levels of the GABA benzodiazepine receptor in alcohol dependency-an 123I-iomazenil SPET study. J Nucl Med 38(Suppl):399.

Lingford-Hughes AR, Acton PD, Gacinovic S, Boddington SJ, Costa DC, Pilowsky LS Ell PJ, Marshall EJ, and Kerwin RW (2000) Levels of gamma-aminobutyric acidbenzodiazepine receptors in abstinent, alcohol-dependent women: preliminary findings from an 123I-iomazenil single photon emission tomography study. Alcohol Clin Exp Res 24:1449-1455.

Lingford-Hughes AR, Acton PD, Gacinovic S, Suckling J, Busatto GF, Boddington SJ, Bullmore E, Woodruff PW, Costa DC, Pilowsky LS, et al (1998) Reduced levels of GABA-benzodiazepine receptor in alcohol dependency in the absence of grey matter atrophy. Br J Psychiatry 173:116-122.

López-Moreno JA, Marcos M, Calleja-Conde J, Echeverry-Alzate V, Bühler KM, Costa-Alba P, Bernardo E, Laso F-J, Rodríguez de Fonseca F, Nadal R, et al. (2015) Histone deacetylase gene expression following binge alcohol consumption in rats and humans. Alcohol Clin Exp Res 39:1939-1950.

Lund IV, Hu Y, Raol YH, Benham RS, Faris R, Russek SJ, and Brooks-Kayal AR (2008) $\mathrm{BDNF}$ selectively regulates $\mathrm{GABA}_{\mathrm{A}}$ receptor transcription by activation of the JAK/STAT pathway. Sci Signal 1:ra9.

Malvaez M, McQuown SC, Rogge GA, Astarabadi M, Jacques V, Carreiro S, Rusche JR, and Wood MA (2013) HDAC3-selective inhibitor enhances extinction of cocaine-seeking behavior in a persistent manner. Proc Natl Acad Sci USA 110: $2647-2652$

Mathelier A, Fornes O, Arenillas DJ, Chen CY, Denay G, Lee J, Shi W, Shyr C, Tan G, Worsley-Hunt R, et al. (2016) JASPAR 2016: a major expansion and update of the open-access database of transcription factor binding profiles. Nucleic Acids Res 44(D1):D110-D115.

Mhatre MC and Ticku MK (1992) Chronic ethanol administration alters gammaaminobutyric acidA receptor gene expression. Mol Pharmacol 42:415-422.

Montpied P, Morrow AL, Karanian JW, Ginns EI, Martin BM, and Paul SM (1991) Prolonged ethanol inhalation decreases gamma-aminobutyric acidA receptor alpha subunit mRNAs in the rat cerebral cortex. Mol Pharmacol 39:157-163.

Moonat S, Sakharkar AJ, Zhang H, Tang L, and Pandey SC (2013) Aberrant histone deacetylase2-mediated histone modifications and synaptic plasticity in the amygdala predisposes to anxiety and alcoholism. Biol Psychiatry 73:763-773.

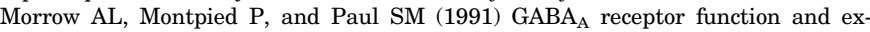
pression following chronic ethanol and barbiturate administration. Ann N Y Acad Sci 625:496-507.

Morrow AL, Suzdak PD, Karanian JW, and Paul SM (1988) Chronic ethanol administration alters $\gamma$-aminobutyric acid, pentobarbital and ethanol-mediated ${ }^{36} \mathrm{Cl}$ uptake in cerebral cortical synaptoneurosomes. J Pharmacol Exp Ther 246: 158-164

Pandey SC, Chartoff EH, Carlezon WA, Jr, Zou J, Zhang H, Kreibich AS, Blendy JA and Crews FT (2005) CREB gene transcription factors: role in molecular mechanisms of alcohol and drug addiction. Alcohol Clin Exp Res 29:176-184. 
Pandey SC, Kyzar EJ, and Zhang H (2017) Epigenetic basis of the dark side of alcohol addiction. Neuropharmacology 122:74-84.

Pandey SC, Ugale R, Zhang H, Tang L, and Prakash A (2008) Brain chromatin remodeling: a novel mechanism of alcoholism. J Neurosci 28:3729-3737.

Ponomarev I, Wang S, Zhang L, Harris RA, and Mayfield RD (2012) Gene coexpression networks in human brain identify epigenetic modifications in alcohol dependence. J Neurosci 32:1884-1897.

Poulter MO, Du L, Weaver ICG, Palkovits M, Faludi G, Merali Z, Szyf M, and Anisman $\mathrm{H}$ (2008) $\mathrm{GABA}_{\mathrm{A}}$ receptor promoter hypermethylation in suicide brain: implications for the involvement of epigenetic processes. Biol Psychiatry 64 645-652.

Romieu P, Host L, Gobaille S, Sandner G, Aunis D, and Zwiller J (2008) Histone deacetylase inhibitors decrease cocaine but not sucrose self-administration in rats. $J$ Neurosci 28:9342-9348.

Sanna E, Mostallino MC, Busonero F, Talani G, Tranquilli S, Mameli M, Spiga S, Follesa P, and Biggio $\mathrm{G}$ (2003) Changes in $\left.\mathrm{GABA}_{\mathrm{A}}\right)$ receptor gene expression associated with selective alterations in receptor function and pharmacology after ethanol withdrawal. J Neurosci 23:11711-11724.

Simon-O'Brien E, Alaux-Cantin S, Warnault V, Buttolo R, Naassila M, and Vilpoux C (2015) The histone deacetylase inhibitor sodium butyrate decreases excessive ethanol intake in dependent animals. Addict Biol 20:676-689.

Taylor C, Nash J, Rich A, Lingford-Hughes A, Nutt D, and Potokar J (2008) Assessment of $\mathrm{GABA}_{\mathrm{A}}$ benzodiazepine receptor $(\mathrm{GBzR})$ sensitivity in patients with alcohol dependence. Alcohol Alcohol 43:614-618.
Thakore PI, D’Ippolito AM, Song L, Safi A, Shivakumar NK, Kabadi AM, Reddy TE, Crawford GE, and Gersbach CA (2015) Highly specific epigenome editing by CRISPR-Cas9 repressors for silencing of distal regulatory elements. Nat Methods 12:1143-1149.

Uusi-Oukari M and Korpi ER (2010) Regulation of $\mathrm{GABA}_{\mathrm{A}}$ ) receptor subunit expression by pharmacological agents. Pharmacol Rev 62:97-135.

Van Lint C, Emiliani S, and Verdin E (1996) The expression of a small fraction of cellular genes is changed in response to histone hyperacetylation. Gene Expr 5:245-253.

Wang Z, Zang C, Cui K, Schones DE, Barski A, Peng W, and Zhao K (2009) Genomewide mapping of HATs and HDACs reveals distinct functions in active and inactive genes. Cell 138:1019-1031.

Wang Z, Zang C, Rosenfeld JA, Schones DE, Barski A, Cuddapah S, Cui K, Roh T-Y, Peng W, Zhang MQ, et al. (2008) Combinatorial patterns of histone acetylations and methylations in the human genome. Nat Genet 40:897-903.

Warnault V, Darcq E, Levine A, Barak S, and Ron D (2013) Chromatin remodelinga novel strategy to control excessive alcohol drinking. Transl Psychiatry 3:e231.

Werner DF, Kumar S, Criswell HE, Suryanarayanan A, Fetzer JA, Comerford CE, and Morrow AL (2011) PKC $\gamma$ is required for ethanol-induced increases in GABA(A) receptor $\alpha 4$ subunit expression in cultured cerebral cortical neurons. J Neurochem 116:554-563.

Address correspondence to: A. Leslie Morrow, 3027 Thurston-Bowles Bldg., CB 7178, Chapel Hill, NC, 27599-7178. E-mail: morrow@med.unc.edu 